\title{
Fabrication and evaluation of surface plasmon resonance optical sensor for heavy metal ions detection
}

\begin{abstract}
Application of surface plasmon resonance (SPR) in sensing heavy metal ions has been emerged since more than a decade ago. A wide range of active layers or recognition elements (e.g. polymer, protein, nanoparticles) have been developed to combine with SPR. The detection limit, sensitivity and selectivity of SPR sensing in heavy metal ions have been improved from time to time, until today. Different advancements on active layers or recognition elements have been discussed in detail and arranged in the order of their chronological evolution. The present study may provide researchers valuable information regarding novel optical sensor for heavy metal ion detection and hence for further research and development.
\end{abstract}

Keyword: Surface plasmon resonance; Optical sensor; Heavy metal ions 